\title{
Selection of the NIR Region for a Regression Model of the Ethanol Concentration in Fermentation Process by an Online NIR and Mid-IR Dual-Region Spectrometer and 2D Heterospectral Correlation Spectroscopy
}

\author{
Takashi NiSHII,* Takuma GenKaWA, ${ }^{*}, * *$ Masahiro WATARI, ${ }^{*}, * * *$ and Yukihiro OzaKI* \\ *School of Science and Technology, Kwansei Gakuin University, 2-1 Gakuen, Sanda 669-1337, Japan \\ **Faculty of Life and Environmental Sciences, University of Tsukuba, 1-1-1 Tennodai, Tsukuba 305-8572, \\ Japan \\ ***Industry Marketing Department, Yokogawa Electric Corporation, 2-9-32 Nakacho, Musashino 180-8750, \\ Japan
}

\begin{abstract}
A new selection procedure of an informative near-infrared (NIR) region for regression model building is proposed that uses an online NIR/mid-infrared (mid-IR) dual-region spectrometer in conjunction with two-dimensional (2D) NIR/mid-IR heterospectral correlation spectroscopy. In this procedure, both NIR and mid-IR spectra of a liquid sample are acquired sequentially during a reaction process using the NIR/mid-IR dual-region spectrometer; the 2D NIR/mid-IR heterospectral correlation spectrum is subsequently calculated from the obtained spectral data set. From the calculated 2D spectrum, a NIR region is selected that includes bands of high positive correlation intensity with mid-IR bands assigned to the analyte, and used for the construction of a regression model. To evaluate the performance of this procedure, a partial least-squares (PLS) regression model of the ethanol concentration in a fermentation process was constructed. During fermentation, $\mathrm{NIR} /$ mid-IR spectra in the $10000-1200 \mathrm{~cm}^{-1}$ region were acquired every $3 \mathrm{~min}$, and a $2 \mathrm{D} \mathrm{NIR} / \mathrm{mid}-\mathrm{IR}$ heterospectral correlation spectrum was calculated to investigate the correlation intensity between the NIR and mid-IR bands. NIR regions that include bands at 4343, 4416, 5778, 5904, and $5955 \mathrm{~cm}^{-1}$, which result from the combinations and overtones of the C-H group of ethanol, were selected for use in the PLS regression models, by taking the correlation intensity of a mid-IR band at $2985 \mathrm{~cm}^{-1}$ arising from the $\mathrm{CH}_{3}$ asymmetric stretching vibration mode of ethanol as a reference. The predicted results indicate that the ethanol concentrations calculated from the PLS regression models fit well to those obtained by high-performance liquid chromatography. Thus, it can be concluded that the selection procedure using the $\mathrm{NIR} /$ mid-IR dual-region spectrometer combined with 2D NIR/mid-IR heterospectral correlation spectroscopy is a powerful method for the construction of a reliable regression model.
\end{abstract}

(Received August 30, 2012; Accepted October 24, 2012; Published December 10, 2012)

\section{Introduction}

In recent years, the developments of spectroscopic process analysis techniques that can reliably monitor and provide deeper understanding of such processes are highly desired. Since nearinfrared (NIR) spectroscopy has various advantages for process monitoring in industrial plants, for instance the usage of optical fibers and non-destructive analysis, it has been recognized as one of the more useful techniques for process monitoring. ${ }^{1-4}$

Prior to process monitoring using NIR spectroscopy in the plants, a regression model of an analyte must be constructed in laboratories. Although many regression methods, for instance, a multiple linear regression method, have been proposed, their predictive accuracy of the model depends on the wavelength selection. A partial least-squares (PLS) regression method builds a regression model from the spectral region, so that the selection of wavelengths is not required. However, there is

† To whom correspondence should be addressed.

E-mail: genkawa.takuma.fm@u.tsukuba.ac.jp increasing evidence indicating, either theoretically or experimentally, that wavelength selection can still significantly refine the performance of full-spectrum calibration techniques. ${ }^{5}$ In other words, the predictive accuracy of the PLS regression model can be raised by identifying informative spectral regions that contain useful information for PLS modeling. ${ }^{5,6}$ Several powerful methods exist for informative regions selection. For example, moving window PLS (MWPLS) regression has been used extensively for informative regions selection in various spectroscopic techniques, such as mid-IR, NIR, and Raman spectroscopy. ${ }^{5,6}$ Although MWPLS regression is a powerful method, it is important to note that those spectral regions are selected based on the sum of the squared residuals. Therefore, the assignment of the NIR band, which is included in the selected region, is argued to validate the selected region. Unfortunately, the assignment of the NIR spectra is often not so straightforward as that of spectra in the mid-IR region.

In the present study a selection method of the informative NIR regions based on the combination of an online NIR/mid-IR dual-region spectrometer and $2 \mathrm{D} \mathrm{NIR} /$ mid-IR heterospectral correlation analysis is proposed. In this method, time-dependent 
NIR/mid-IR spectra of a liquid sample during a reaction process are acquired by the online NIR/mid-IR dual-region spectrometer The 2D NIR/mid-IR heterospectral correlation spectrum is subsequently calculated from the obtained spectral data set to explore an informative NIR region that includes bands of high positive correlation intensity with mid-IR bands assigned to the analyte. The online NIR/mid-IR dual-region spectrometer was recently developed, and facilitates the sequential measurement of both NIR (10000 - $\left.4000 \mathrm{~cm}^{-1}\right)$ and mid-IR $\left(5000-1200 \mathrm{~cm}^{-1}\right)$ spectra of a liquid sample, ${ }^{7}$ which are required to calculate the 2D NIR/mid-IR heterospectral correlation spectrum. Generalized 2D correlation spectroscopy and its heterospectral correlation analysis were proposed by Noda, ${ }^{8-11}$ and many studies concerning the assignments of the NIR band by using 2D NIR/mid-IR heterospectral correlation spectroscopy have been reported. ${ }^{12-17}$ The proposed method applies this capability of 2D NIR/mid-IR heterospectral correlation analysis on NIR band assignments to the selection of the informative NIR region for regression model construction.

One of the advantages of the proposed selection method is that the NIR regions are determined indirectly based on the assignment of the mid-IR band of the analyte, and thus the selected regions are validated from a chemical standpoint. Another advantage is that knowledge obtained by mid-IR spectroscopy can be utilized in the construction of a regression model using NIR spectroscopy. Yet another one is that no quantitative data about the analyte obtained by chemical analysis methods is required, because the absorption intensities of the mid-IR band arising from the analyte are used as quantitative data in the $2 \mathrm{D} \mathrm{NIR/mid-IR} \mathrm{heterospectral} \mathrm{correlation} \mathrm{analysis.}$ Therefore, the feasibility of process monitoring by NIR spectroscopy can be evaluated from the presence of the informative NIR region without measuring a reference value. This feature is more beneficial, particularly when the chemical analysis method for the reference data is time-consuming.

The performance of the proposed method is evaluated using the ethanol fermentation process. Fermentation monitoring has been investigated using NIR, mid-IR, and Raman spectroscopy because of its importance in several fields, such as food, pharmaceutical, and biological sciences and technologies. ${ }^{718-22}$ In our previous work, ethanol fermentation was monitored with the online NIR/mid-IR dual-region spectrometer, and principal-component analysis revealed that the detection of the end-point in the fermentation can be performed by using either transmission NIR and attenuated total reflection (ATR) mid-IR spectra based on the same chemical changes: relative changes in the concentrations of glucose and ethanol. ${ }^{7}$ In the present study, time-dependent NIR/mid-IR spectra of the fermentation solution were acquired, and NIR bands assigned to the $\mathrm{CH}$ group were detected from the 2D NIR/mid-IR heterospectral correlation spectrum calculated from the obtained spectra. NIR regions that include these NIR bands were used for PLS modeling, and the results indicate that the proposed selection method has superior performance in terms of effective selection of ethanol bands.

\section{Materials and Methods}

\section{Spectral measurement}

Immobilized baker's yeast $(40 \mathrm{~mL}$ for the training sample set and $80 \mathrm{~mL}$ for the test sample set) was added to a $200-\mathrm{mL}$ of liquid culture medium (200 $\mathrm{g} \mathrm{L}^{-1}$ glucose, $10 \mathrm{~g} \mathrm{~L}^{-1}$ peptone, and $5 \mathrm{~g} \mathrm{~L}^{-1}$ yeast extract) to prepare the sample for fermentation process monitoring. A sample was put into a special beaker fitted with a glass-jacket with a magnetic stirring bar, and the temperature of the sample was maintained at $30^{\circ} \mathrm{C}$ during fermentation by controlling the water temperature in the jacket. NIR and mid-IR spectra in the $10000-1200 \mathrm{~cm}^{-1}$ region of the fermentation solution during the fermentation process were measured by the NIR/mid-IR dual-region spectrometer. ${ }^{7}$ This spectrometer consists of a halogen lamp, a Fourier transform NIR/mid-IR interferometer, a light-path switching device, a transmission immersion NIR probe with $1 \mathrm{~mm}$ optical pathlength, an ATR immersion mid-IR probe with ZnSe internal reflection element, and detectors for NIR mid-IR regions, facilitating the acquisition of time-dependent NIR/mid-IR spectra of a liquid sample during a reaction process by switching the light path leading to the probes. Details of this spectrometer were described in our previous paper. ${ }^{7}$

Firstly, both NIR and mid-IR probes were set in an empty beaker, and background spectra were measured in the NIR and mid-IR region by using air. The liquid culture medium was subsequently poured into the beaker. Spectral measurements were started $1 \mathrm{~min}$ after adding the immobilized yeast to the liquid culture medium. All spectra were collected about every $3 \mathrm{~min}$ at a $8-\mathrm{cm}^{-1}$ spectral resolution, and 128 scans were co-added to ensure an adequate signal-to-noise ratio.

\section{Reference method}

Reference data of the ethanol concentrations of the fermentation solution during fermentation were measured by a HPLC (JASCO Model AS-2055puls and PU-2089Plus). The HPLC system was equipped with a column heater, auto sampler, a computer controller, and a JASCO Model RI-1531 refractive index detector. A COSMOSIL 5 $\mathrm{C}_{18}$-PAQ Packed Column $(250 \mathrm{~mm} \times 4.6 \mathrm{~mm})$ was used, and the temperature of the column was set at $25^{\circ} \mathrm{C}$. The mobile phase consisted of a solution of $0.5 \%\left(4.4 \times 10^{-6} \mathrm{~N}\right)$ trifluroacetic acid in water at a flow rate of $1.0 \mathrm{ml} \mathrm{min}{ }^{-1}$. During fermentation, a few milliliters of the fermentation solution were sampled by a syringe and immediately provided for the HPLC measurement. The concentration range of ethanol was $0-83.0 \mathrm{~g} \mathrm{~L}^{-1}$ for the training sample set (43 specimens) and $0-75.8 \mathrm{~g} \mathrm{~L}^{-1}$ for the test sample set (30 specimens).

\section{Spectral analysis}

The spectral data files were converted into the Unscrambler software format (Ver. 9.2: CAMO AS, Trondheim, Norway). Before a 2D NIR/mid-IR heterospectral correlation analysis and the PLS regression analysis, we investigated a pretreatment method to reduce the effect of baseline fluctuations in the raw spectra on these analyses. In this respect, second-derivative and multiplicative scatter correction (MSC) treatments are well-known approaches. In the present study, we used the MSC treatment for 2D NIR/mid-IR heterospectral correlation analysis because possible artifacts caused by ripples in the second derivative spectra might make the interpretation of the 2D correlation maps difficult. For developing the PLS regression model, the second-derivative treatment with Savitzky-Golay smoothing (7 points, 2 nd orders) was employed to reduce the effects of any baseline fluctuation and overlapping of bands on the prediction results.

The 2D NIR/mid-IR heterospectral correlation spectra were calculated from MSC treated NIR (4900-4250 and $\left.6200-5700 \mathrm{~cm}^{-1}\right)$ and mid-IR $\left(3500-2700 \mathrm{~cm}^{-1}\right)$ spectra by using 2DShige software (Dr. Shigeaki Morita). Unshaded and shaded regions in a $2 \mathrm{D}$ correlation spectrum indicate positive and negative correlation intensities, respectively. The PLS regression model of the ethanol concentration in the fermentation solution was constructed using the Unscrambler software. After 


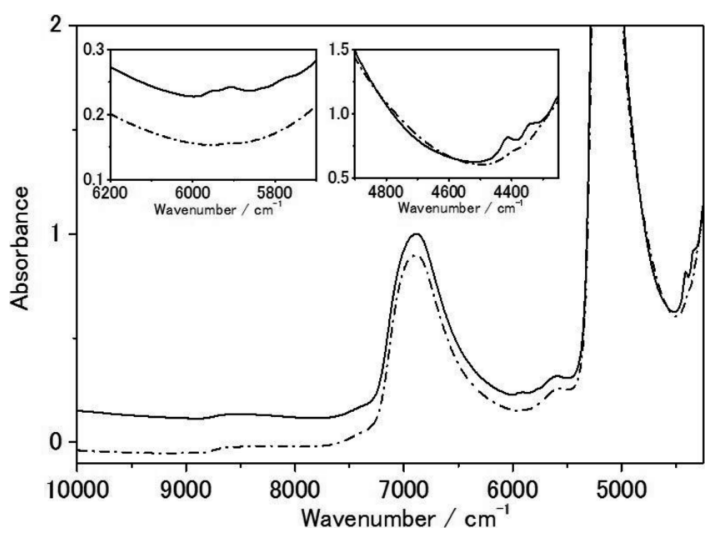

Fig. 1 NIR spectra of the fermentation solution at the beginning (dashed line) and end (solid line) of the fermentation process.

selection of the NIR region, 2nd derivative and mean-centering treatments, root mean square error of cross-validation were calculated using the full-cross validation method to determine the optimum number of latent variables (LVs), based on the occurrence of a significant difference in the explained variance of the ethanol concentration. Using the prediction samples, the determination coefficient $\left(R^{2}\right)$ and the root-mean-square-error of prediction (RMSEP) were calculated.

\section{Results and Discussion}

\section{NIR spectra of the solution during fermentation}

Figure 1 shows NIR spectra in the $10000-4200 \mathrm{~cm}^{-1}$ region measured at the beginning and the end of ethanol fermentation using a training sample set. The inset shows an enlargement of the $6200-5700$ and $4800-4500 \mathrm{~cm}^{-1}$ region. It is noted that the baseline shifts upward with an increase in the scatting intensity due to an increase in the biomass. An increase in the ethanol content and a decrease in the glucose content were observed in the $6200-5700$ and $4900-4250 \mathrm{~cm}^{-1}$ region. In the latter region, the bands at 4416 and $4340 \mathrm{~cm}^{-1}$ increase and the broad feature in the $4850-4500 \mathrm{~cm}^{-1}$ region decreases. In the former region, the baseline increases over the entire NIR region, while an intensity increase can be seen for the $5985-5870 \mathrm{~cm}^{-1}$ region and the band at $5790 \mathrm{~cm}^{-1}$. On the other hand, in the mid-IR spectra a band at $2985 \mathrm{~cm}^{-1}$ increases during fermentation (Fig. 2). According to the research on ethanol-water binary mixtures by Burikov et al. ${ }^{23}$ and the mid-IR spectra measured preliminarily depicting that no band arising from glucose, peptone, and yeast extract appears at $2985 \mathrm{~cm}^{-1}$, the mid-IR band at $2985 \mathrm{~cm}^{-1}$ is assigned to the $\mathrm{CH}_{3}$ asymmetric stretching vibration mode of ethanol. The spectral changes during fermentation using the test sample set were almost similar to those of using the training sample set, except for the fermentation time, because the amount of immobilized yeast of the test sample set was twice that of the training sample set.

Selection of informative NIR regions by using $2 D$ NIR/mid-IR heterospectral correlation analysis

We calculated the 2D NIR/mid-IR heterospectral correlation spectrum by using the $3500-2800 \mathrm{~cm}^{-1}$ region containing the $2985 \mathrm{~cm}^{-1}$ band to explore the NIR regions suitable for constructing a PLS regression model of the ethanol concentration.

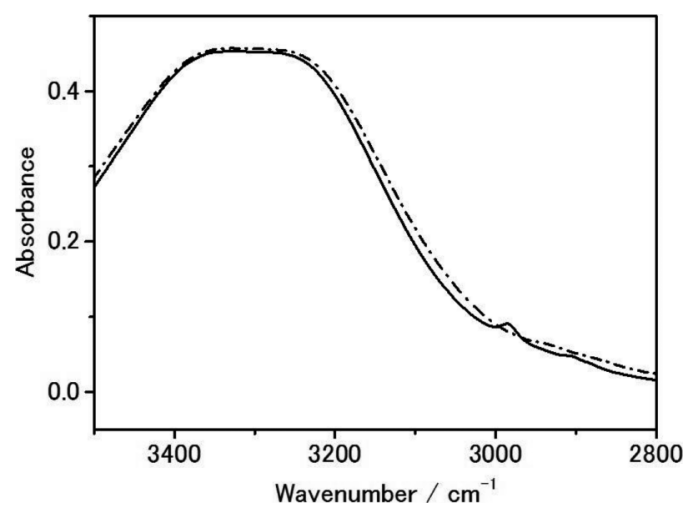

Fig. 2 Mid-IR spectra in the $3500-2800 \mathrm{~cm}^{-1}$ region of the fermentation solution at the beginning (dashed line) and end (solid line) of the fermentation process.
Figure 3 shows the 2D NIR/mid-IR heterospectral correlation spectra of the fermentation solution. Mean spectra are provided at the top and right side of the $2 \mathrm{D}$ spectra. It was found that the NIR bands at 4340 and $4416 \mathrm{~cm}^{-1}$ have a relatively high positive correlation intensity with the $2985 \mathrm{~cm}^{-1}$ band (Fig. 3(b)). Therefore, these bands arise from the combinations of the $\mathrm{C}-\mathrm{H}$ vibrations of ethanol. Moreover, a negative cross peak is observed in the $4850-4500 \mathrm{~cm}^{-1}$ region. The existence of a negative cross peak between the ethanol band at $2985 \mathrm{~cm}^{-1}$ and those in the $4850-4500 \mathrm{~cm}^{-1}$ region suggests that the bands in the $4850-4500 \mathrm{~cm}^{-1}$ region arise from glucose. Similarly, one can assign the bands in the region of $5985-5870 \mathrm{~cm}^{-1}$ and at $5790 \mathrm{~cm}^{-1}$ to the overtones or combinations of the $\mathrm{C}-\mathrm{H}$ stretching vibrations of ethanol, and the bands in the region of $6170-5990 \mathrm{~cm}^{-1}$ to the overtone of the O-H group of glucose (Fig. 3(a)). Based on these assignments, NIR regions that include bands at 4343, 4416, 5778, 5904, and $5955 \mathrm{~cm}^{-1}$ were selected as the informative regions, and used for PLS regression models of ethanol concentration.

\section{PLS regression models using different NIR regions}

To evaluate the performance of the proposed selection procedure, PLS regression models using different NIR regions were constructed. The prediction results obtained from these models are summarized in Table 1. For the developments of all models, second-derivative spectra were used, except for Model VI. The NIR regions used for Model I and Model II were selected by reference to the $2 \mathrm{D} \mathrm{NIR} /$ mid-IR heterospectral correlation spectra shown in Fig. 3. Model III and Model IV employed the NIR region of $6300-4250 \mathrm{~cm}^{-1}$, which includes bands arising from $\mathrm{C}-\mathrm{H}$ groups of ethanol and glucose and the O-H group of glucose. Model V and Model VI were constructed by using the full NIR region, and Model VII was constructed by using second-derivative mid-IR spectra in the $3500-2800 \mathrm{~cm}^{-1}$ region. The results concerning the optimum number of LVs mean that, except for Model VI, the first LV of each model contains sufficient information to describe a change in the ethanol concentration during the fermentation process. In addition, the observed high $R^{2}$ and small RMSEP indicate that all PLS regression models, including Model VI, can accurately predict the ethanol concentration during fermentation within a few LVs.

To validate the constructed models, the PLS loadings of the first LV of each model were interpreted. Figures 4(a) and 4(b) show the loading plots of the first LV of Model III and 

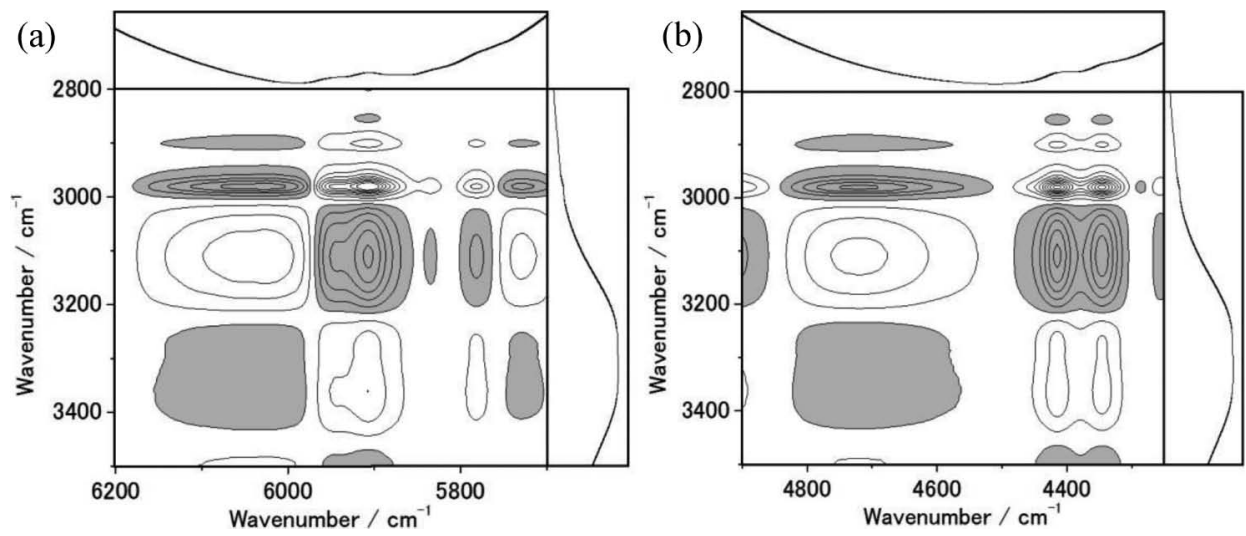

Fig. 3 Synchronous 2D NIR/mid-IR heterospectral correlation spectra calculated from the time-dependent spectra of the fermentation solution.
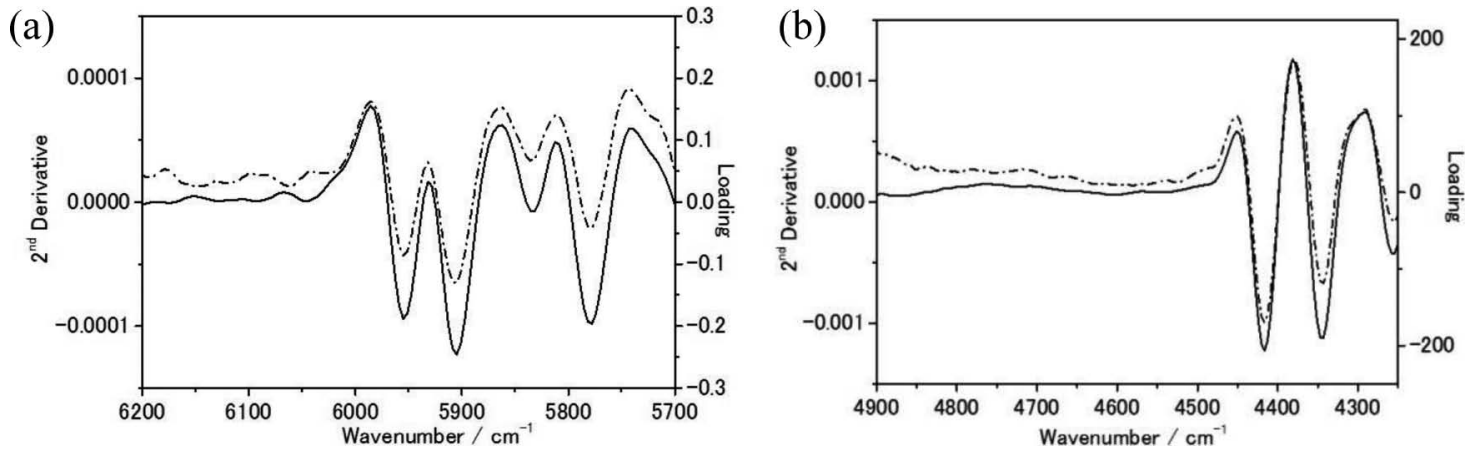

Fig. 4 Loading plots of the first LV of (a) Model III and (b) Model IV. Dashed lines indicate the second-derivative spectra of a $35 \mathrm{~g} \mathrm{~L}^{-1}$ aqueous ethanol solution.

Table 1 Calibration results of PLS regression models using different NIR regions

\begin{tabular}{|c|c|c|c|c|c|c|c|}
\hline Model & I & II & III & IV & $\mathrm{V}$ & VI & VII \\
\hline Pretreatment method & 2nd der. & 2nd der. & 2nd der. & 2nd der. & 2nd der. & - & 2nd der. \\
\hline Spectral region $/ \mathrm{cm}^{-1}$ & $\begin{array}{r}5985-5870, \\
5812-5742\end{array}$ & $4450-4300$ & $6300-5700$ & $4900-4250$ & $\begin{array}{c}10000-6400 \\
4900-4200\end{array}$ & $10000-4200$ & $3500-2800$ \\
\hline Number of LVs & 1 & 1 & 1 & 1 & 1 & 2 & 1 \\
\hline$R^{2}$ & 0.997 & 0.997 & 0.997 & 0.997 & 0.997 & 0.982 & 0.997 \\
\hline RMSEP/g L ${ }^{-1}$ & 2.2 & 1.7 & 2.2 & 1.7 & 1.7 & 4.9 & 1.7 \\
\hline
\end{tabular}

Model IV, respectively. The second-derivative spectra of a $35 \mathrm{~g} \mathrm{~L}^{-1}$ aqueous ethanol solution are also presented. Larger loadings were obtained at 4343, 4416, 5778, 5904, and $5955 \mathrm{~cm}^{-1}$, and it was found that these bands correspond to bands assigned to the $\mathrm{C}-\mathrm{H}$ group of ethanol. It is therefore reasonable to assume that Model III and Model IV can be used to monitor changes in the ethanol concentration during the fermentation process. In addition, these bands also correspond to the bands used in Model I and Model II, which display a relatively high positive correlation intensity with the mid-IR band assigned to the $\mathrm{CH}_{3}$ asymmetric stretching vibration modes of ethanol. These results reveal that the $2 \mathrm{D} \mathrm{NIR} /$ mid-IR heterospectral correlation spectrum calculated from the time-dependent NIR/mid-IR spectra of the fermentation solution can provide us with the informative NIR regions used in the regression models.

Score and loading plots of the first LV of Model V are shown in Figs. 5(a) and 5(b). The scores increase with the fermentation time, and larger loadings are obtained in the $4500-4200 \mathrm{~cm}^{-1}$ region, which is similar to the loadings obtained with Model II and Model IV. Thus, the estimation of the ethanol concentration by Model $\mathrm{V}$ also occurs through the band arising from the $\mathrm{C}-\mathrm{H}$ group of ethanol. Although the score plot of the first LV of Model VI shown in Fig. 6(a) is similar to that of Model V, the corresponding loading plot shown in Fig. 6(b) is quite different from that of Model V, indicating that the first LV of Model VI reflects a different phenomenon. In the $10000-5500 \mathrm{~cm}^{-1}$ region, the loading becomes larger at higher wavenumbers. Absorption intensities in this region increase with the fermentation time because of an upward shift in the baseline, with the upward shift being larger at higher wavenumbers (Fig. 1). This behavior is typical for loading plots showing scattering phenomena. Small, yet meaningful, features in the $7400-7000$ and $6200-5700 \mathrm{~cm}^{-1}$ regions seem to show a shift of the $\mathrm{O}-\mathrm{H}$ band to higher wavenumbers, and a relative change 

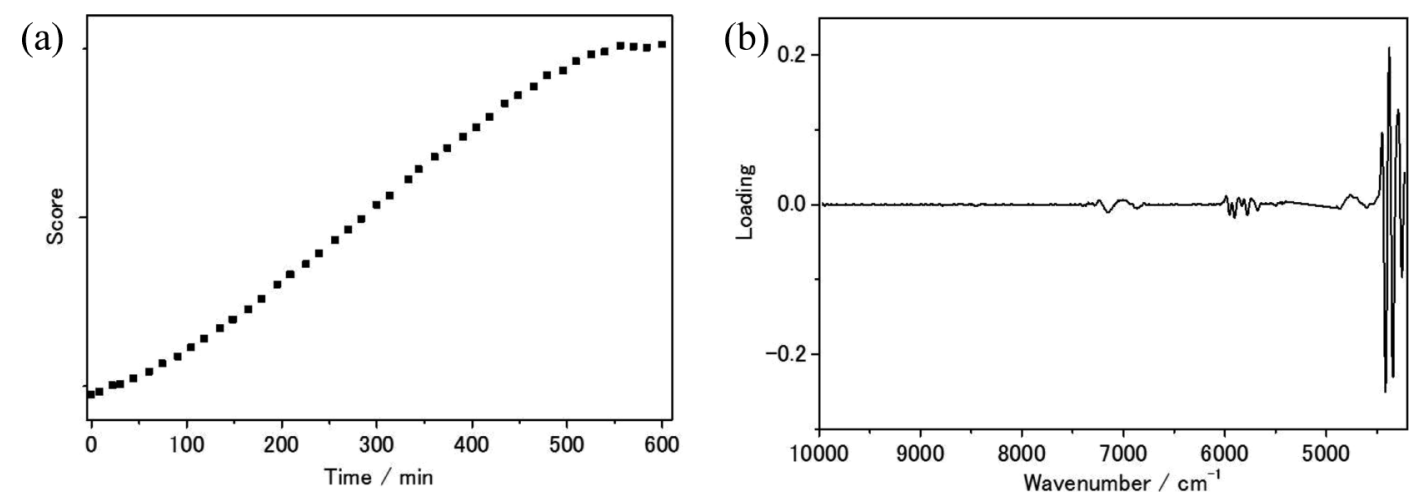

Fig. 5 (a) Score and (b) loading plots of the first LV of Model V constructed by using raw spectra in the $9966-6400 \mathrm{~cm}^{-1}$ region.
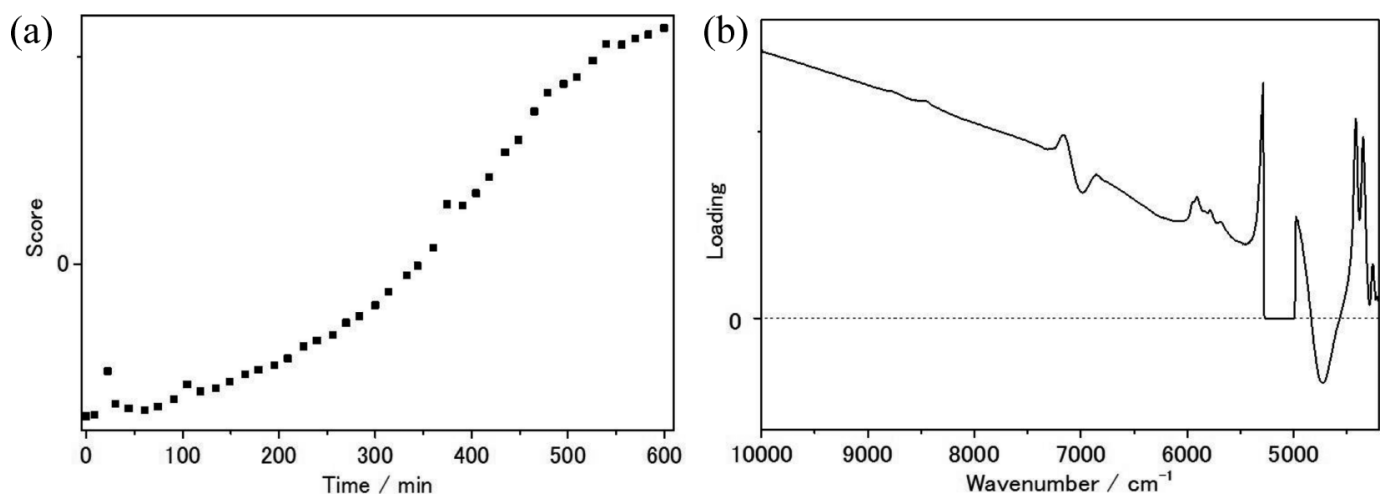

Fig. 6 (a) Score and (b) loading plots of the first LV of Model VI constructed by using raw spectra in the $10000-4200 \mathrm{~cm}^{-1}$ region.
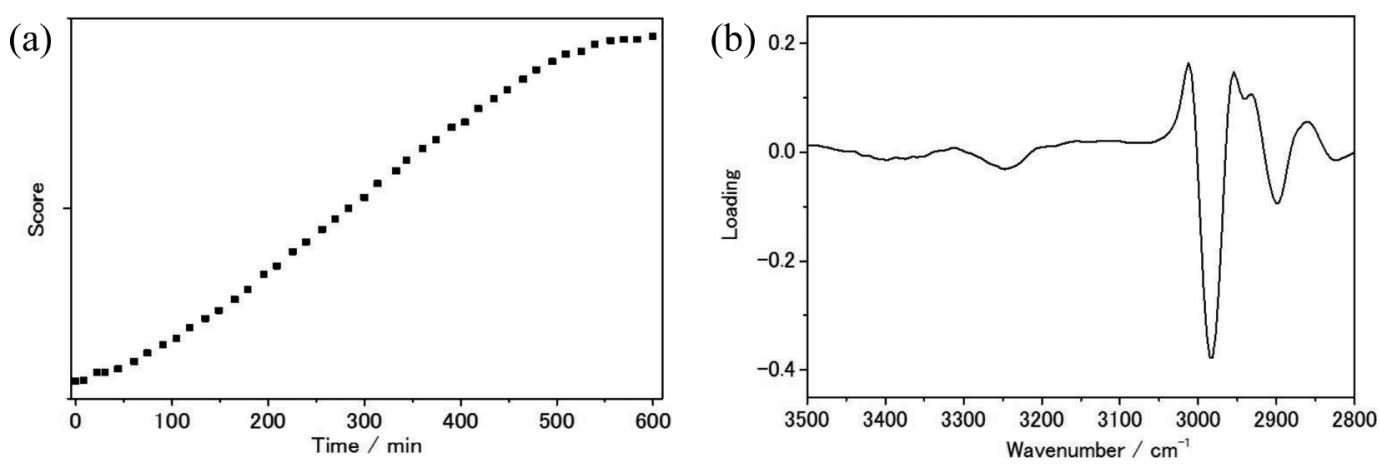

Fig. 7 (a) Score and (b) loading plots of the first LV of Model VII constructed by using the second derivative spectra in the $3500-2800 \mathrm{~cm}^{-1}$ region

in the ethanol and glucose concentrations, but these are obscured by the baseline upward shift. In the lower wavenumber region, the scattering effect becomes smaller, and we can observe a shift of the band at $5400 \mathrm{~cm}^{-1}$ due to O-H vibrations, while a useful loading plot is obtained in the $4900-4200 \mathrm{~cm}^{-1}$ region, showing the relative change in the glucose and ethanol concentrations. These results confirm that Model VI estimates the ethanol concentration mainly based on a baseline upward shift caused by scattering.

The change in the scattering intensity arises from the increase in biomass in the fermentation solution. It is therefore very likely that the increase in the scattering intensity caused by fermentation yields a high $R^{2}$ and a small RMSEP, as is exemplified in the test set (Table 1). The present results demonstrate that an evaluation of a regression model should not only take into account $R^{2}$ and RMSEP, but also the interpretation of the scores and the loadings. Figure 7 depicts the scores and the loadings for the first LV of Model VII obtained by the second-derivative mid-IR spectra. The scores show a similar variation to that shown in Fig. 5(a), while the loadings yield a large peak at $2985 \mathrm{~cm}^{-1}$. It is therefore clear that Model VII directly detects the concentration change of ethanol. The $R^{2}$ and RMSEP are almost identical to that of Model II, Model IV, and Model V, which employ the NIR region. 


\section{Conclusions}

In the present work, we have proposed a new selection method of the informative NIR regions for the construction of a regression model using the combination of an online NIR/mid-IR dual-region spectrometer and 2D NIR/mid-IR heterospectral correlation spectroscopy. This method enables the selection of a suitable NIR region for developing the regression model by reference against an intensity change of a mid-IR band, whose assignment has been established, even if no reference data of the analyte exists. By using the online NIR/mid-IR dual-region spectrometer we measured the time-dependent NIR/mid-IR spectra of a fermentation solution, and used 2D NIR/mid-IR heterospectral correlation analysis to search for NIR bands that are strongly correlated with the mid-IR ethanol band. A PLS regression model that was developed by using a NIR region that contained the NIR bands showed very promising prediction results with a $R^{2}$ of 0.997 and a RMSEP of $1.7 \mathrm{~g} \mathrm{~L}^{-1}$ when employing only the first LV. Thus, it is concluded that the proposed method is very useful in effectively determining the informative NIR regions suitable for the construction of regression models.

\section{Acknowledgements}

The study was financially supported by the Innovation Promotion Program from the New Energy and Industrial Technology Development Organization (NEDO), Ministry of Economy, Trade and Industry, Japan. The authors thank Prof. Hidetoshi Yamada (Kwansei Gakuin University) for the quantitative HPLC analysis of ethanol and Prof. Takuo Yano (Hiroshima City University) for his kind advice in the fermentation experiments.

\section{References}

1. Y. Ozaki, Anal. Sci., 2012, 28, 545.

2. K. A. Lee, "Near-Infrared Spectroscopy in Food Science and Technology", ed. Y. Ozaki, W. F. McCluer, and A. A. Christy, 2006, Chap. 9, Wiley Interscience, New York, 361.

3. M. B. Simpson, "Process Analytical Technology:
Spectroscopic Tools and Implementation Strategies for the Chemical and Pharmaceutical Industries", ed. K. A. Bakeev, 2nd ed., 2010, John Wiley \& Sons, Ltd., Chichester, UK, 107.

4. M. Watari, Opt. Rev., 2010, 17, 317.

5. J. H. Jiang, R. J. Berry, H. W. Siesler, and Y. Ozaki, Anal. Chem., 2002, 74, 3555.

6. Y. P. Du, Y. Z. Liang, J. H. Jiang, R. J. Berry, and Y. Ozaki, Anal. Chim. Acta, 2004, 501, 183.

7. T. Genkawa, M. Watari, T. Nishii, and Y. Ozaki, Appl. Spectrosc., 2012, 66, 773.

8. I. Noda, Appl. Spectrosc., 1993, 47, 1329.

9. I. Noda, A. E. Dowrey, and C. I. Marcott, Appl. Spectrosc., 1993, 47, 1317.

10. I. Noda and Y. Ozaki. "Two-dimensional Correlation Spectroscopy-Applications in Vibrational and Optical Spectroscopy_”, 2004, John Wiley \& Sons Ltd., Chichester, UK, 1.

11. I. Noda, Anal. Sci., 2007, 23, 139.

12. T. Amari and Y. Ozaki, Macromolecules, 2002, 35, 8020.

13. B. Czarnik-Matusewicz, S. Pilorz, and J. P. Hawranek, Anal. Chim. Acta, 2005, 544, 15.

14. R. A. Cocciardi, A. A. Ismail, Y. Wang, and J. Sedman, J. Agric. Food Chem., 2006, 54, 6475.

15. S. Watanabe, I. Noda, and Y. Ozaki, J. Mol. Struct., 2008, 883-884, 173.

16. S. Watanabe, I. Noda, and Y. Ozaki, Polymer, 2008, 49, 774 .

17. A. Aït Kaddour, M. Mondet, and B. Cuq, J. Cereal Sci., 2008, 48, 678.

18. V. R. Kondepati and H. M. Heise, Curr. Trends Biotechnol. Pharm., 2008, 2, 117.

19. R. Nagarajan, A. Gupta, R. Mehrotra, and M. M. Bajaj, J. Autom. Methods Manage. Chem., 2006, 2006, 1.

20. A. G. Cavinato, D. M. Mayes, Z. Ge, and J. B. Callis, Anal. Chem., 1990, 62, 1977.

21. M. Blanco, A. C. Peinado, and J. Mas, Biotechnol. Bioeng., 2004, 88, 536.

22. V. Di Egidio, N. Sinelli, G. Giovanelli, A. Moles, and E. Casiraghi, Eur. Food Res. Technol., 2010, $230,947$.

23. S. Burikov, T. Dolenko, S. Patsaeva, Y. Starokurov, and V. Yuzhakov, Mol. Phys., 2010, 108, 2427. 